Bundesgesundheitsbl - Gesundheitsforsch Gesundheitsschutz 2004 · 47:1115-1123 DOI 10.1007/s00103-004-0931-3

๑) Springer Medizin Verlag 2004

\section{Genetische Polymorphismen (Sequenzvariationen) von Fremdstoff-metabolisierenden Enzymen und ihre Bedeutung in der Umweltmedizin}

\author{
Mitteilung der Kommission „Methoden \\ und Qualitätssicherung in der Umweltmedizin"
}

\section{Vorbemerkung}

Immer wieder wird beobachtet, dass in einer Gruppe von Menschen, die unter vergleichbaren Bedingungen leben und gegenüber bestimmten Umweltfaktoren exponiert sind, nur einige Individuen im Zusammenhang mit diesen Expositionen von gesundheitlichen Auswirkungen betroffen sein können. In der Auseinandersetzung mit der Umwelt und den aufgenommenen Fremdstoffen spielen die Fremdstoff-metabolisierenden Enzyme (FME) eine große Rolle. So genannte „Polymorphismen“ oder Sequenzvariationen dieser Enzyme können, wie aus Studien zu Arzneimittelnebenwirkungen und Unglücksfällen bekannt, für erhebliche interindividuelle Unterschiede im Fremdstoffmetabolismus verantwortlich sein.

Sequenzvariationen treten häufig im menschlichen Genom auf, die SNPs (Single Nucleotide Polymorphisms) werden mit einer Häufigkeit von 1 zu ca. 1.000 Basenpaaren (bp) beobachtet. Unter einem „Polymorphismus“ wird das Auftreten einer Genvariation in einer Population verstanden, definitionsgemäß muss die Allelfrequenz für das variante Allel $>1 \%$ in der Bevölkerung sein, anderenfalls wird von einer Mutation gesprochen. Eindeutiger ist der Begriff, „Sequenz- variation", der sich aber noch nicht vollständig durchgesetzt hat. Der Begriff Mutation sollte nicht in diesem Zusammenhang verwendet werden, da dieser Begriff in der Toxikologie in anderem Kontext benutzt wird. Die wichtigsten Typen von Sequenzvariationen werden nachfolgend kurz dargestellt.

\section{Sequenzvariationen}

Folgende Arten von Sequenzvariationen bzw. Polymorphismen lassen sich unterscheiden:

- SNP (Single Nucleotide Polymorphisms, z. B. NAT2),

- Insertions- und Deletionspolymorphismen (z. B. GSTT1), - Multiplikationen (z. B. CYP2D6).

Die häufigsten Sequenzvariationen sind die Einzelnukleotidpolymorphismen oder Single-Nucleotide-Polymorphisms (SNP), bei denen es zum Austausch eines Nukleotides im DNA-Molekül kommt. Es wird von einem SNP auf 200-1.00o bp im menschlichen Genom ausgegangen [1]. Die SNPs sind nicht gleichmäßig im gesamten Genom verteilt. Für die funktionelle Auswirkung sind die Art und die Position des Nukleotidaustausches von Bedeutung. Liegt der SNP im kodierenden
Bereich einer Gensequenz, kann das einen Aminosäureaustausch zur Folge haben; da aber viele Aminosäuren von mehreren Basentripletts kodiert werden, hat nicht jeder Basenaustausch in der DNA zwingend einen Aminosäureaustausch im Protein zur Folge. Die Aminosäuresubstitution kann die Stabilität und die Funktionalität des Proteins beeinflussen, bei Enzymen sind neben Aktivitätsverminderungen auch -erhöhungen beobachtet worden.

Unter einer Insertion oder Deletion versteht man den Einbau oder den Verlust von mindestens einem Nukleotid. Beispiele für Deletionsvariationen sind die Glutathion-S-Transferasen GSTM1 [2] und GSTT1 [3], bei denen große Teile des jeweiligen Gens fehlen. Als Folge kann keine enzymatische Aktivität bestimmt werden, wenn das Gen auf beiden Allelen deletiert ist. Es wurden auch Multiplikationen des gesamten Gens beobachtet. Dies kann zur erheblichen Erhöhung der metabolischen Kapazität führen (z. B. $\mathrm{CYP}_{2} D 6$ ). Eine ausführliche Monographiensammlung zu Methoden der Bestimmung von Sequenzvariationen wird demnächst von der DFG-Arbeitsgruppe „Analysen im biologischen Material" veröffentlicht [4]. Die darin beschriebenen Methoden sind validiert, und es wird dort ausführlich auf Fragen der Qualitätssicherung und der Bedeutung dieser Untersuchungen eingegangen. 


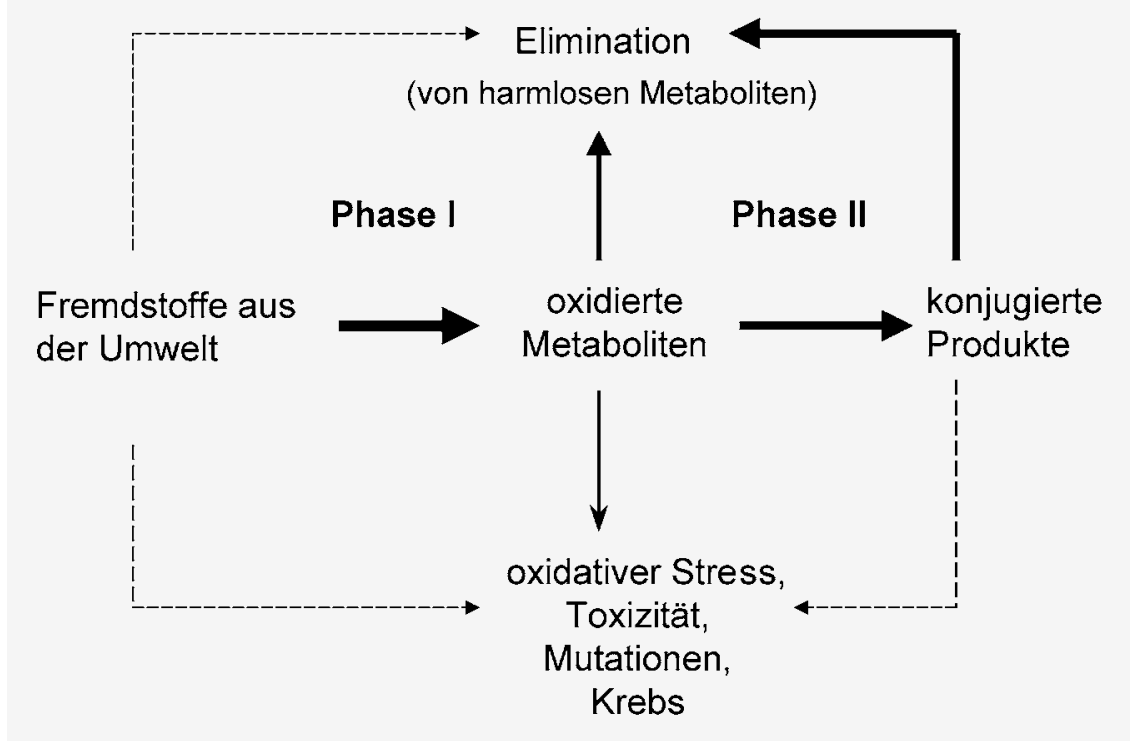

Abb. $1 \Delta$ Der Phase-I- und Phase-II-Metabolismus führt einerseits zur Entstehung von reaktiven Intermediaten und andererseits zur Elimination von Fremdstoffen [5]

\section{Fremdstoffmetabolismus und individuelle Disposition}

Der menschliche Organismus kann sich mittels vielfältiger Enzymsysteme der vielen Umweltsubstanzen entledigen, die oral, dermal oder inhalativ resorbiert werden. Viele dieser Fremdstoffe sind so lipophil, dass sie ohne weitere metabolische Modifikationen nicht eliminiert werden können. Dies gilt auch für viele endogen synthetisierte Stoffe, z. B. Hormone, die nach erfolgreicher Signalübermittlung ebenfalls ausgeschieden werden müssen. Die beteiligten Enzymsysteme zeichnen sich durch ein bemerkenswert breites Substratspektrum aus und werden in PhaseI- und Phase-II-Enzyme unterschieden (- Abb. 1): Phase-I-Reaktionen umfassen im Wesentlichen oxidative Reaktionen, die zur Bildung von polaren und reaktiven Metaboliten führen. Die wichtigste Enzymgruppe stellen hier die Cytochrom-P450-abhängigen Monooxygenasen dar (CYP). Die Metaboliten der Phase-I-Enzyme dienen dann den Konjugationsreaktionen der Phase II als Substrate und werden dadurch im Allgemeinen hydrophiler und eliminierbar. In der Regel entstehen bei den Reaktionen der Phase II biologisch inaktive Metaboliten. Enzyme der Phase II, zu denen die Glutathion-S-Transferasen (GST), die N-Acetyltransferasen (NAT), die UDP-Glukurono- syltransferasen (UGT) und die Sulfotransferasen gehören, können auch geeignete Fremdstoffe ohne vorhergehende Prozessierung in der Phase I metabolisieren, diese Möglichkeit wurde in der $\bullet$ Abb. 1 aus Gründen der Übersichtlichkeit nicht dargestellt. Die aus didaktischer Sicht eingeführte Unterscheidung des Fremdstoffmetabolismus in Phase I=Giftung und Phase II=Entgiftung ist eine einprägsame, aber drastisch simplifizierte Sichtweise, die einer differenzierten Betrachtung, wie für die Umweltmedizin erforderlich, hinderlich ist.

Aus der Umwelt werden Fremdstoffe wie Inhaltsstoffe von Lebensmitteln, Arzneimitteln, Industriechemikalien (Arbeitsstoffe) und viele andere Verbindungen aufgenommen. Lipophile Substanzen lassen sich praktisch kaum unverändert eliminieren. Durch die Enzyme der Phase I erfolgt eine Oxidation, an die sich die Kopplungsreaktionen der Phase II anschließen. Die Dicke der Pfeile stellt eine semiquantitative Schätzung der Bedeutung der Stoffwechselwege dar.

Der größere Teil der Substanzen wird durch den Fremdstoffmetabolismus inaktiviert, es können allerdings auch biologisch aktive Substanzen gebildet werden; dies geschieht meist über oxidative Reaktionen. So wurde geschätzt, dass etwa $25 \%$ aller Arzneistoffe und umweltrelevanten Schadstoffe ihre unerwünschten Wirkun- gen erst nach metabolischer Aktivierung durch FME entfalten [6]. Neben Proteinaddukten konzentriert sich das toxikologische Interesse vor allen Dingen auf die Bildung von DNA-Addukten: Zwar werden die meisten DNA-Addukte durch zelleigene Reparaturenzyme entfernt, dennoch können einige Addukte persistieren und bei der nächsten Zellteilung zu Ablesefehlern führen (Mutationen) und damit den ersten Schritt im Prozess der chemisch induzierten Kanzerogenese darstellen [7]. Bei einer Bewertung der Metabolisierungsschritte muss aber immer berücksichtigt werden, dass auch der Phase-I-Metabolismus überwiegend inaktive Produkte generiert (• Abb. 1).

Wird eine Substanz ausschließlich oder zum überwiegenden Teil von einem einzelnen FME umgesetzt, dann können funktionell relevante Sequenzvariationen dieses Enzyms erhebliche Auswirkungen haben. Umweltbedingte Einflüsse können neben den Sequenzvariationen über eine Induktion oder eine Inhibition dieses Enzyms erhebliche Folgen haben. Zur Bedeutung dieser Phänomene sei auf Übersichtsartikel verwiesen [5].

\section{Fremdstoff-metabolisierende Enzyme}

\subsection{Cytochrome P450}

Die Cytochrom P450-abhängigen Monooxygenasen sind die wichtigste Enzymgruppe der Phase I, sie bestehen aus dem Häm-Protein Cytochrom $\mathrm{P}_{450}$ und der Cytochrom-P450-Reduktase: Für die Substratspezifität sind die Cytochrome $\mathrm{P} 450$ verantwortlich. Inzwischen sind für den Menschen mehr als 30 verschiedene Cytochrome $\mathrm{P} 450$ charakterisiert, die am Fremdstoffmetabolismus beteiligt sind [8] (http://www.imm.ki.se/CYPalleles/). Eine Übersicht über die wichtigsten $\mathrm{P}_{450}$ Formen wird in $\bullet$ Tabelle 1 gegeben. Dort wird auch für jedes Enzym auf die OMIMDatenbank des NCBI hingewiesen (http:// www.ncbi.nlm.nih.gov). In dieser Datenbank finden sich in erster Linie Angaben zur Genetik, die Qualität der toxikologischen Bewertung ist variabel. Die Einträge werden regelmäßig aktualisiert. Eine ausführliche Darstellung wurde von Schulz vorgelegt [5]. 
Tabelle 1

Polymorphe Cytochrome P450

\begin{tabular}{|c|c|c|c|c|c|c|}
\hline $\begin{array}{l}\text { Gen } \\
\text { (MIM \#) }\end{array}$ & Induzierbar & Induktoren & $\begin{array}{l}\text { Charakteristische Substrate } \\
\text { und Beispiele }\end{array}$ & $\begin{array}{l}\text { Metabolische } \\
\text { Relevanz für } \\
\text { Umweltschadstoffe }\end{array}$ & $\begin{array}{l}\text { Umweltmedizinische } \\
\text { Relevanz der } \\
\text { Sequenzvariation }\end{array}$ & $\begin{array}{l}\text { Indikation zur } \\
\text { Analyse der } \\
\text { Sequenzvariation }\end{array}$ \\
\hline $\begin{array}{l}\text { CYP1A1 } \\
(108330)\end{array}$ & Ja & $\begin{array}{l}\text { Dioxine, } \\
\text { Zigaretten- } \\
\text { rauch }\end{array}$ & $\begin{array}{l}\text { Polyzyklische KW } \\
\text { (z. B. Benz[a]pyren) }\end{array}$ & Ja & Keine & Keine \\
\hline $\begin{array}{l}\text { CYP1A2 } \\
(124060)\end{array}$ & Ja & $\begin{array}{l}\text { Dioxine, } \\
\text { Zigaretten- } \\
\text { rauch }\end{array}$ & $\begin{array}{l}\text { Homo- und heterozyklische } \\
\text { aromatische Amine }\end{array}$ & Ja & Keine & Keine \\
\hline $\begin{array}{l}\text { CYP1B1 } \\
(601771)\end{array}$ & Ja & Dioxine & $\begin{array}{l}\text { Polyzyklische KW } \\
\text { (z. B. Benz[a]pyren) }\end{array}$ & Ja & Keine & Keine \\
\hline $\begin{array}{l}\text { CYP2A6 } \\
(122720)\end{array}$ & $\mathrm{Ja}$, in vitro & Phenobarbital & Nikotin, Nitrosamine & Gering & Keine & Keine \\
\hline $\begin{array}{l}\text { CYP2C9 } \\
(601130)\end{array}$ & Ja & Rifampicin & Benz[a]pyren, Arzneistoffe & Gering & Keine & Keine \\
\hline $\begin{array}{l}\text { CYP2C19 } \\
(124020)\end{array}$ & Ja & Rifampicin & Arzneistoffe & Keine/gering & Keine & Keine \\
\hline $\begin{array}{l}\text { CYP2D6 } \\
(124030)\end{array}$ & Nein & & $\begin{array}{l}\text { Nitrosamine, viele } \\
\text { Arzneistoffe }\end{array}$ & Gering & Keine & Keine \\
\hline $\begin{array}{l}\text { CYP2E1 } \\
(124040)\end{array}$ & Ja & $\begin{array}{l}\text { Ethanol, } \\
\text { Isoniazid }\end{array}$ & $\begin{array}{l}\text { Ethanol, Nitrosamine, } \\
\text { Vinylchlorid, Benzol }\end{array}$ & $\mathrm{Ja}$ & Keine & Keine \\
\hline $\begin{array}{l}\text { CYP3A4 } \\
(124010)\end{array}$ & Ja & $\begin{array}{l}\text { Steroide, } \\
\text { Rifampicin } \\
\text { u. a. }\end{array}$ & $\begin{array}{l}\text { Aflatoxin B1 u. a. Mykotoxine, } \\
\text { viele Arzneistoffe } \\
\text { (z. B. Cyclosporin) }\end{array}$ & Gering & Keine & Keine \\
\hline $\begin{array}{l}\text { CYP3A5 } \\
(605325)\end{array}$ & Offen & & Arzneistoffe & Keine/gering & Keine & Keine \\
\hline
\end{tabular}

Weitere Informationen zu den einzelnen Enzymen sind im Internet unter http://www.ncbi.nlm.nih.gov mit der MIM-Nummer im Bereich OMIM (Online Mendelian Inheritance in Man) zu finden. Anmerkung zum Verständnis der Spalten: Unter "charakteristischen Substraten und Beispielen" werden relevante Beispiele genannt, für einige Enzyme sind praktisch nur Arzneistoffe als Substrate beschrieben. Die ", metabolische Relevanz für Umweltschadstoffe" bezieht sich auf die Fähigkeit des Enzyms, umweltrelevante Schadstoffe zu metabolisieren. Unter "umweltmed. Relevanz der Sequenzvariationen" wird die Bedeutung der Sequenzvariationen für die Modulierung der Enzymaktivität bei umweltmedizinischen Fragestellungen bewertet. Zuletzt fasst die Spalte „Indikation zur Analyse der Sequenzvariation" die Einschätzung der Kommission zum Einsatz dieser diagnostischen Möglichkeiten in der Umweltmedizin zusammen.

Die Untersuchung von Sequenzvariationen der Cytochrome P45o kann nach derzeitigem Kenntnisstand nicht empfohlen werden. Bisher wurden keine Studien vorgelegt, die einen individualmedizinischen Nutzen der Kenntnisse der Sequenzvariationen der Cytochrome P450 im umweltmedizinischen Bereich belegen.

\subsection{Glutathion-S-Transferasen}

Die Glutathion-S-Transferasen (GST) sind Enzyme der Phase II und konjugieren elektrophile Ausgangsverbindungen oder Metaboliten mit L-Glutathion. In der Regel wirkt dieser Stoffwechselschritt biologisch inaktivierend [9]. Es wurden allerdings auch hier toxikologisch relevan- te Ausnahmen beschrieben, wie z. B. das Trichlorethen [10]: Das Glutathionkonjugat des Trichlorethens wird von Peptidasen weiter metabolisiert, das entstandene $S$-(1,2-Dichlorvinyl)-L-Cystein wird dann von der $\beta$-Lyase in der Niere zu einem hochreaktiven Thioketen umgesetzt. Daher sollten GST nicht pauschal als detoxifizierende Enzyme bezeichnet werden. Zurzeit sind Sequenzvariationen für einen großen Teil der GST bekannt. Es handelt sich um die GSTA1 und GSTA2, die GSTM1, die GSTM 3 , die GSTP1, die GSTT1 und die GSTZ1 (• Tabelle 2). Eine ausführliche Darstellung findet sich $u$. a. bei Schulz (2003) [5], hier soll nur auf die häufig diskutierten Enzyme GSTM1 und GSTT1 eingegangen werden.
Die GSTM1 wird hauptsächlich in der Leber exprimiert, das Enzym wurde aber auch in anderen Organen nachgewiesen [11]. Es konjugiert u. a. 4-Nitrobenzylchlorid, Chlordinitrobenzol, Styroloxid und Epoxide der polyzyklischen Aromaten mit Glutathion und hat daher große Bedeutung als entgiftendes Enzym. Die GSTM1 kann nicht induziert werden. Es liegen 3 Sequenzvariationen vor, relevant ist aber nur der Deletionspolymorphismus, der bei homozygoten Merkmalsträgern den Verlust der enzymatischen Aktivität zur Folge hat [3]. In europäischstämmigen Bevölkerungen sind - ebenso wie in asiatischen Populationen - etwa 50\% defizient für die GSTM1. Es gibt jedoch klare ethnische Unterschiede, so liegt dieser An- 


\section{Empfehlung des Robert Koch-Instituts}

Tabelle 2

Polymorphe Phase-II-Enzyme

\begin{tabular}{|c|c|c|c|c|}
\hline $\begin{array}{l}\text { Gen } \\
\text { (MIM \#) }\end{array}$ & $\begin{array}{l}\text { Charakteristische } \\
\text { Substrate und Beispiele }\end{array}$ & $\begin{array}{l}\text { Metabolische } \\
\text { Relevanz für } \\
\text { Umweltschad- } \\
\text { stoffe }\end{array}$ & $\begin{array}{l}\text { Umweltmedizi- } \\
\text { nische Relevanz } \\
\text { der Sequenz- } \\
\text { variation }\end{array}$ & $\begin{array}{l}\text { Indikation } \\
\text { zur Analyse } \\
\text { der Sequenz- } \\
\text { variation }\end{array}$ \\
\hline $\begin{array}{l}\text { GSTA1 } \\
\text { (138359) }\end{array}$ & $\begin{array}{l}\text { Stickstofflostverbindungen, } \\
\text { Dibenzpyrendiolepoxide }\end{array}$ & Gering & Keine & Keine \\
\hline $\begin{array}{l}\text { GSTM1 } \\
(138350)\end{array}$ & $\begin{array}{l}\text { 4-Nitrobenzylchlorid, } \\
\text { Epoxide der polyzyklischen } \\
\text { Aromaten }\end{array}$ & $\mathrm{Ja}$ & Keine & Keine \\
\hline $\begin{array}{l}\text { GSTM3 } \\
(138390)\end{array}$ & Wasserstoffperoxid & Bisher keine & Keine & Keine \\
\hline $\begin{array}{l}\text { GSTP1 } \\
(134660)\end{array}$ & $\begin{array}{l}\text { Acrolein, Ethacrynsäure, } \\
\text { Chlordinitrobenzol }\end{array}$ & Bisher gering & Keine & Keine \\
\hline $\begin{array}{l}\text { GSTT1 } \\
(600436)\end{array}$ & $\begin{array}{l}\text { Dichlormethan, } \\
\text { Halomethane, Ethylenoxid, } \\
\text { Haloethane }\end{array}$ & $\mathrm{Ja}$ & Keine & Keine \\
\hline $\begin{array}{l}\text { NAT1 } \\
(108345)\end{array}$ & $\begin{array}{l}\text { Aromatische Amine, } \\
\text { p-Aminosalizylsäure, } \\
\text { p-Aminobenzoesäure }\end{array}$ & Bisher gering & Keine & Keine \\
\hline $\begin{array}{l}\text { NAT2 } \\
(243400)\end{array}$ & $\begin{array}{l}\text { Aromatische Amine, } \\
\text { Sulfamethazin }\end{array}$ & $\mathrm{Ja}$ & Keine & Keine \\
\hline $\begin{array}{l}\text { SULT1A1 } \\
(171150)\end{array}$ & $\begin{array}{l}\text { Phenole, 4-Nitrophenol, } \\
\text { Arylverbindungen }\end{array}$ & Bisher gering & Keine & Keine \\
\hline $\begin{array}{l}\text { SULT1A3 } \\
(600641)\end{array}$ & Phenole, Katecholamine & Bisher gering & Keine & Keine \\
\hline $\begin{array}{l}\text { SULT2A1 } \\
(125263)\end{array}$ & $\begin{array}{l}\text { Steroide, } \\
\text { Hydroxyacetylaminofluoren }\end{array}$ & Bisher gering & Keine & Keine \\
\hline $\begin{array}{l}\text { UGT1A1 } \\
(191740)\end{array}$ & Bilirubin, 1-Naphtol & Bisher gering & Keine & Keine \\
\hline $\begin{array}{l}\text { UGT1A6 } \\
(606431)\end{array}$ & $\begin{array}{l}\text { 3-Hydroxy-Benzo[a]pyren, } \\
\text { 2-Naphtylamin }\end{array}$ & Gering & Keine & Keine \\
\hline
\end{tabular}

teil bei Personen afrikanischer Herkunft bei etwa $27 \%$ [12]. Für eine Vielzahl von malignen Erkrankungen wurden Zusammenhänge zwischen dem GSTM1-Genotyp und der Erkrankung postuliert. Durch Metaanalysen konnten diese Vermutungen bislang nur für den Blasenkrebs und den negativen GSTM1-Genotyp belegt werden [13]. Inkonsistente Ergebnisse ergaben sich für den Zusammenhang zwischen negativem GSTM1-Genotyp und Kopf-HalsTumoren. Von 21 ausgewerteten Studien zeigten 8 Studien ein erhöhtes Risiko [14]. Ebenfalls inkonsistent waren die Assoziationen zwischen GSTM1-Genotyp und Kolonkarzinom [15]. Eine Metaanalyse von 43 Fall-Kontroll-Studien konnte kein erhöhtes Lungenkrebsrisiko für den nega- tiven GSTM1-Genotyp ermitteln [16]. Zwischen dem negativen GSTM1-Genotyp und dem Ovarialkarzinom wurde ebenfalls keine Assoziation gefunden [17].

Die GSTM1-Sequenzvariation ist aufgrund der Interaktion von Umwelteinwirkungen (z. B. Zigarettenrauch) und Organismus von wissenschaftlichem Interesse, allerdings sind Zusammenhänge zwischen malignen Erkrankungen und dem negativem Genotyp nur für den Blasenkrebs im Hochdosisbereich bei langjähriger Einwirkung gesichert. Eine generelle Testung der Bevölkerung kann nicht empfohlen werden, und ein individualmedizinischer Nutzen bei umweltmedizinischen Fragestellungen ist derzeit nicht erkennbar.
Die GSTT1 wird in erheblichem MaBe in der menschlichen Leber, der Niere und den Erythrozyten exprimiert [17, 18]. Darüber hinaus wurde das Enzym auch noch in anderen Geweben wie Dünndarm, Gehirn und Prostata nachgewiesen [11]. Die GSTT1 produziert, wie die anderen GST auch, überwiegend inaktive Metaboliten. $\mathrm{Zu}$ den Substraten gehören u. a. Epoxy-3-(p-Nitrophenoxy-)Propan, Dichlormethan, Chlormethan und Brommethan [11, 18]. Andererseits kann das Enzym aber auch Substanzen wie Dichlormethan zu genotoxischen Intermediaten metabolisieren. Es liegen keine Hinweise auf eine Induzierbarkeit und Inhibierbarkeit der GSTT1 in vivo vor. Bereits die partielle Gendeletion hat [4] - ebenso wie bei der GSTM1 - einen Aktivitätsverlust des Enzyms bei homozygoten Trägern zur Folge.

Untersuchungen zum Zusammenhang zwischen der länger bekannten GSTT1Gendeletion und der enzymatischen Umsetzung der Substrate Methylchlorid oder Dichlormethan wurden sowohl in Erythrozyten als auch in der Leber durchgeführt und haben dort eindeutig zeigen können, dass eine GSTT1-abhängige Enzymaktivität nur vorhanden ist, wenn das GSTT1-Gen vollständig vorhanden ist [19]. Wie bei der GSTM1 wurden auch für das Vorkommen des $\operatorname{GSTT1}_{1}{ }^{*}$-Genotyps deutliche Unterschiede in verschiedenen ethnischen Gruppen festgestellt, so liegt der Anteil der homozygoten Träger des $\mathrm{GSTT1}_{1}{ }^{*} \mathrm{O}$-Allels unter Europäern bei etwa $20 \%$, während er unter Asiaten bei fast $50 \%$ liegt [12].

Die GSTT1-Deletion kann bei akzidenteller, hoher Exposition gegenüber Substanzen wie Methylbromid die Schwere der Vergiftungssymptomatik beeinflussen [20]. Mögliche Zusammenhänge zwischen malignen Erkrankungen und dem GSTT1-Polymorphismus sind in einer Reihe von molekularepidemiologischen Studien untersucht worden (Zusammenfassung in [5]). Beispielhaft wird hier auf postulierte Zusammenhänge zwischen der Entwicklung eines myelodysplastischen Syndroms (MDS) und dem Fehlen des funktionalen GSTT1-Gens hingewiesen; die Autoren vermuteten eine fehlende Metabolisierungskapazität, die zu einer Entstehung dieser Erkrankung beitra- 
gen könnte [21]. Diesem Befund folgten weitere Studien anderer Arbeitsgruppen, die diesen Zusammenhang nicht bestätigen konnten $[\mathbf{2 2}, \mathbf{2 3}]$. Inkonsistente Ergebnisse gab es für den Zusammenhang zwischen negativem GSTT1-Genotyp und Kopf-Hals-Tumoren, 6 von 14 Studien wiesen auf ein erhöhtes Risiko hin [14]. Ähnlich inkonsistent waren die Assoziationen mit dem Kolonkarzinom und dem Ovarialkarzinom $[15,17]$.

Die Deletionsvariante der GSTT1 hat zwar aufgrund der Interaktion von Umwelteinwirkungen (z. B. Zigarettenrauch) und Organismus eine wissenschaftliche Bedeutung, da das Enzym sowohl biologisch inaktivierende als auch aktivierende Eigenschaften haben kann; ein individualmedizinischer Nutzen bei umweltmedizinischen Fragestellungen ist derzeit jedoch nicht erkennbar.

\subsection{N-Acetyltransferasen}

Auch die N-Acetyltransferasen (NAT) sind Enzyme der Phase II. Beim Menschen sind 2 NAT (NAT1 und NAT2) beschrieben, die die $\mathrm{N}$ - und O-Acetylierung von Fremdstoffen katalysieren. Die Substratspezifität der beiden Enzyme überlappt erheblich [24], eine eindeutige Abgrenzung der beiden NAT voneinander gestaltet sich daher schwierig. Toxikologische Relevanz besitzen die beiden Enzyme wegen ihrer Fähigkeit, aromatische und heterozyklische Amine entweder zu aktivieren oder $\mathrm{zu}$ inaktivieren.

Die NAT2 ist das FME, für das zuerst ein Polymorphismus im Zusammenhang mit der Einnahme von Medikamenten beschrieben wurde. Bereits in den 5oer-Jahren des 20. Jahrhunderts wurde in „schnelle" und „langsame Acetylierer" unterschieden. Das Enzym wird hauptsächlich in der Leber und nur in sehr geringem Maß extrahepatisch exprimiert. Es werden Substrate wie das Sulfamethazin bevorzugt von der NAT2 metabolisiert [24].

Die Ursache für den phänotypisch beschriebenen Polymorphismus liegt in verschiedenen Sequenzvariationen, die zum teilweisen Aktivitätsverlust des Enzyms führen. Für das NAT2-Gen wurden 13 SNP beschrieben, von denen 9 einen Aminosäureaustausch zur Folge haben [25]. Durch Kombination dieser SNP kann es zu einer Vielzahl von varianten Allelen kommen. Eine Übersicht über die derzeit mehr als 26 varianten NAT2Allele findet sich in der Datenbank http:// www.louisville.edu/medschool/pharmacology/NAT.html. In den mediterranen Ländern sind nur etwa 10\% der Bevölkerung „schnelle Acetylierer“, in Mitteleuropa sind es dann schon etwa $40 \%$ der Bevölkerung, während in Japan mehr als 90\% der Population „schnelle Acetylierer" sind [6].

Die toxikologische und arbeitsmedizinische Relevanz des phänotypisch charakterisierten NAT2-Polymorphismus wird durch ältere Studien bei Arbeitern mit Blasenkrebs unterstützt, wo unter den Blasenkrebsfällen mehr langsame Acetylierer gefunden wurden: Bei Betrachtung einer Subgruppe von Chemiearbeitern aus der Farbstoff verarbeitenden Industrie waren 22 von 23 untersuchten Blasenkrebspatienten langsame Acetylierer [26]. Auch in einem größeren Kollektiv von Beschäftigten in der chemischen Industrie ist diese Häufung der ,langsamen Acetylierer" unter den Patienten mit Blasenkrebs gefunden worden $[27,28]$. Metaanalysen von Blasenkrebsstudien ergaben bisher nur eine geringe statistische Risikoerhöhung, wobei eine Heterogenität zwischen den Studien auffiel [29, 30]. Werden aber noch Umweltfaktoren wie das Rauchen oder eine berufliche Exposition gegenüber Arylaminen berücksichtigt, dann verstärkt sich das Risiko für Blasenkrebs geringfügig [31].

Im Gegensatz dazu gibt es Hinweise auf eine Erhöhung des Risikos für schnelle Acetylierer beim Lungenkrebs: In einer großen Studie wurde ein Zusammenhang zwischen NAT2-Genotyp und dem Lungenkrebs beschrieben [32]. Es wurde ein erhöhtes Krebserkrankungsrisiko für homozygote schnelle Acetylierer festgestellt; dies traf insbesondere für die Gruppe von Patienten mit einem Zigarettenkonsum in der Größenordnung von mehr als 20 „Packjahren“ (ein Packjahr beinhaltet den täglichen Konsum einer Packung Zigaretten in einem Jahr) zu. Vor einer abschließenden Bewertung sollte auch hier die Bestätigung in nachfolgenden Studien abgewartet werden. Widersprüchliche Assoziationen wurden für den schnellen Acetyliererstatus und Kolonkarzinom beobachtet.
Lang und Kollegen beschrieben als Erste eine Assoziation zwischen dem schnellen Acetyliererstatus, der phänotypisch bestimmt wurde, und dem Risiko, am Kolonkarzinom zu erkranken [33]. Diese statistische Assoziation konnte aber in groß angelegten Studien nicht bestätigt werden, sodass aktuelle Metaanalysen zum Schluss kommen, dass der schnelle Acetyliererstatus keinen Risikofaktor für die Entwicklung eines Kolonkarzinoms ausmacht [34].

Ein individualmedizinischer Nutzen einer möglichen Untersuchung bei umweltmedizinischen Fragestellungen ist derzeit nicht erkennbar.

\subsection{Andere Phase-II-Enzyme}

Auch bei anderen Phase-II-Enzymen wie Sulfotransferasen und UDP-Glukuronosyltransferasen (UGT) wurden Sequenzvariationen beschrieben, hier sei auf Übersichtsarbeiten verwiesen $[5,35,36]$.

\subsection{Ethnische Unterschiede in der Verteilung der Sequenz- variationen}

Es wurden erhebliche ethnische Unterschiede für das Auftreten von Sequenzvariationen bei verschiedenen FME beobachtet. Als Beispiel sind hier verschiedene Studien für die GSTT1 und die GSTM1 zusammengefasst, die zeigen, dass z. B. in chinesischen Populationen der Anteil der GSTT1-negativen Individuen wesentlich höher ist als in Mitteleuropa. Für die GSTM1 sind vergleichbare Unterschiede zwischen Chinesen und Europäern nicht beobachtet worden. Andererseits ist der Anteil der GSTM1-negativen Individuen unter Afrikanern wesentlich niedriger als unter Europäern. Am niedrigsten ist der Anteil GSTM1-negativer Personen unter den australischen Ureinwohnern. Die Gründe für diese unterschiedlichen Verteilungen in verschiedenen Ethnien sind immer noch unklar. Der Einfluss von Faktoren des Lebensstils und der Umwelt wird zwar diskutiert, es bleibt aber noch zu klären, welche Faktoren es im Einzelnen sein können. Neben den GST sind auch für die Cytochrome $\mathrm{P} 450$ und die NAT erhebliche Unterschiede in verschiedenen Ethnien beschrieben worden. 


\section{Auswirkung der Sequenzvariationen und Kombinationswirkungen}

Es wurde bereits erwähnt, dass sowohl Sequenzvariationen als auch Induktionsund Inhibitionsphänomene vor allem dann eine besondere Rolle spielen, wenn ein Fremdstoff ausschließlich oder überwiegend über ein einzelnes FME metabolisiert wird. Die Sequenzvariation eines Enzyms für sich hat ebenso wenig Krankheitswert wie die Augenfarbe. Nur wenn eine bestimmte Sequenzvariation vorliegt und zusätzlich eine entsprechend hohe Exposition gegenüber der kritischen Substanz erfolgt, dann kann sich eine entsprechende schädigende Wirkung manifestieren. Es sind nur wenige Krankheiten bekannt, bei denen ein Enzym einen so zentralen Stellenwert im Organismus besitzt, dass einer vererbbaren vollständigen Inaktivität des Enzyms (z. B. UGT1A1 und Criggler-Najjar-Syndrom) allein schon Krankheitswert zukommt.

Der größere Teil der Substanzen, gegenüber denen wir aus der Umwelt exponiert sind, wird allerdings über eine Vielzahl unterschiedlicher Phase-I- und Phase-II-Reaktionen bzw. -Enzyme umgesetzt. Daraus resultiert einerseits eine Reihe von Möglichkeiten, wie konkurrierende Reaktionen ein vielfältiges Metabolitenmuster erzeugen (Bsp.: Metabolisierung des Benz[a]pyrens). Andererseits ist es nahe liegend, die Kombination der Sequenzvariationen von Phase-I- und Phase-II-Enzymen in wissenschaftlichen Studien auf Gruppenbasis weiter zu untersuchen. Die Kombinationen müssen sich aber am Stoffwechselweg der jeweils untersuchten Fremdstoffe orientieren und dürfen nicht beliebig zusammengestellt werden. Ein gutes Beispiel sind hierzu die Studien zu den FME CYP1A1 und GSTM1 bei Patienten mit Lungenkrebs. Die Patienten waren langjährige Raucher, und es wurde die Frage gestellt, ob bei diesen $\mathrm{Pa}$ tienten die $C Y P_{1} A_{1}$-Variante (die eine bessere Induzierbarkeit aufweisen soll und damit eine höhere Bildung von toxischen Intermediaten ermöglichen könnte) im Zusammenwirken mit der GSTM1-Defizienz der Entgiftung dieser Verbindungen auffällig überrepräsentiert wäre. In japanischen Studien waren diese Zusammen- hänge signifikant [36]. Studien in Populationen europäischer Abstammung (im angelsächsischen Schrifttum als „caucasians" bezeichnet) waren weniger eindeutig aufgrund der wesentlich niedrigeren Allelfrequenzen für die entsprechenden CYP1A1-Varianten (s. auch [5]).

\subsection{Häufigkeit der Phase-I- und Phase-II-Sequenzvariationen}

Bei Betrachtung der bereits beschriebenen Sequenzvariationen der verschiedenen FME fällt auf, dass der Anteil der Personen europäischer Herkunft mit einem inaktiven oder weniger aktiven Phänotyp (bzw. einem Genotyp, der diesen Phänotyp bewirkt) bei den Phase-II-Enzymen GSTM1, GSTT1 und NAT2 zwischen $15 \%$ und $60 \%$ liegt und somit sehr groß ist. Wie eingangs erwähnt, sind die Phase-II-Enzyme im Wesentlichen inaktivierende Enzyme, daher liegt die Vermutung nahe, dass das Fehlen eines dieser Enzyme einen Nachteil in der Auseinandersetzung des Organismus (Individuums) mit reaktiven Metaboliten und einen wichtigen Suszeptibilitätsfaktor für toxikologisch relevante Endpunkte, wie z. B. eine Tumorentstehung, darstellen könnte. Es gibt jedoch für beide Fälle Beispiele, sowohl dass die höhere Aktivität eines Enzyms (z. B. NAT2) auch ein höheres Krankheitsrisiko zur Folge haben kann, als auch, dass das Fehlen einer Enzymaktivität vor bestimmten Krankheitserscheinungen bei Vergiftungen schützen kann (z. B. GSTT1). Bei den Cytochromen P450 sind hingegen die Sequenzvariationen seltener: Hier liegt der Anteil der Personen mit negativem bzw. weniger aktivem $\mathrm{Ge}$ no-/Phänotyp für die Formen $\mathrm{CYP}_{2} \mathrm{~A} 6$, $\mathrm{CYP}_{2} \mathrm{C} 9, \mathrm{CYP}_{2} \mathrm{C}_{19}$ und $\mathrm{CYP}_{2} \mathrm{D} 6$ zwischen $2 \%$ und $10 \%$.

\section{2 Überlegungen zum Krebsrisiko}

Es muss deutlich unterschieden werden zwischen der Betrachtung des individuellen und des kollektiven Risikos. Dies lässt sich z. B. anhand der GST veranschaulichen; hier liegen viele Untersuchungen zu Zusammenhängen zwischen der GSTM1-Sequenzvariation und malignen Erkrankungen vor. Eine Reihe von Untersuchungen zeigte unter Patienten mit be- stimmten malignen Erkrankungen eine Häufung der Individuen mit GSTM1-negativem Genotyp. Allerdings lag die Erhöhung des individuellen Risikos bei Vorliegen des GSTM1-negativen Genotypes in der Regel nur bei einem Faktor von etwa zwei [38]. Im Vergleich zu Änderungen in bestimmten Tumorsuppressorgenen, die vielfache Steigerungen des individuellen Krebsrisikos zur Folge haben, stellt das eine geringe Erhöhung dar. Bei Betrachtung der Auswirkungen auf große Kollektive muss wiederum berücksichtigt werden, dass die GSTM1-Defizienz in Europa etwa 50\% der Bevölkerung betrifft und die GSTT1-Defizienz zwischen 10 und 30\%, während die Veränderungen der Tumorsuppressorgene selten sind. Damit haben die Sequenzvariationen der GST eine gewisse Bedeutung für die Beurteilung eines Risikos für die Gesamtpopulation, weil viele Individuen betroffen sind.

Werden die Studien der letzten Jahre betrachtet, dann fallen die Heterogenität der Ergebnisse und die - in der Regel schwachen Assoziationen auf [39]. Es ist nicht ungewöhnlich, dass eine erste FallKontroll-Studie das Risiko einer Erkrankung im Zusammenhang mit einer Sequenzvariation eines FME überschätzt, während die folgenden größer angelegten Studien diesen Zusammenhang nicht bestätigen können [40]. Nicht nur für Assoziationsstudien im Bereich der FME wurde darauf hingewiesen, dass häufig die erste Studie eine starke Assoziation feststellt und in hochrangigen Zeitschriften publiziert wird, während diese Assoziationen mit der Veröffentlichung von weiteren Studien und dem Anwachsen der verfügbaren Daten schwächer wird oder gar ganz verschwindet [41]. Es sollte daher von Seiten des interessierten Umweltmediziners mit dem nötigen Sachverstand an (sensationell klingende) Veröffentlichungen oder Marketingpapiere herangegangen werden.

In den Metaanalysen und gesammelten Analysen wurden einige Assoziationen für die GSTM1-Deletion und den langsamen Acetyliererstatus gefunden. Die Erwartung, eine Genvariante im Fremdstoffmetabolismus zu finden, die als $\mathrm{Ri}$ sikofaktor für Krebs mit einem gewissen Grad an Wahrscheinlichkeit (der Gewiss- 
heit) benannt werden kann und eine Reihenuntersuchung der Bevölkerung sowie Präventionsmaßnahmen rechtfertigen würde, wurde durch die vorliegenden Daten enttäuscht [42]. Positive Korrelationen dienten und dienen der Hypothesengenerierung zu möglichen Risikofaktoren der Krebsentstehung. In Zukunft müssen die Fragestellungen der Studien sorgfältiger ausgearbeitet werden und die Expositionsfaktoren besser berücksichtigt werden.

\subsection{Umweltrelevante Chemikalien und Risikoabschätzungen}

In der Toxikologie können Untersuchungen von Sequenzvariationen der FME im Zusammenhang mit Beschreibungen der Exposition gegenüber Arbeitsstoffen oder umweltrelevanten Chemikalien zu einer genaueren Ermittlung von Risiken dienen. Überlegungen zur Bedeutung der Sequenzvariationen der FME in der Arbeitsmedizin wurden bereits anderenorts veröffentlicht [43]. Die ethische Problematik der Untersuchung von Sequenzvariationen bei Arbeitnehmern wurde u. a. in der Enquete-Kommission des Bundestages diskutiert [44]. Es sind hier u. a. Konflikte zwischen dem Interesse des Arbeitnehmers an einer Klärung möglicher Gesundheitsrisiken und dem damit in Verbindung stehenden möglichen Verlust des Arbeitsplatzes vorstellbar.

Im Bereich der Umweltmedizin liegt das Ausmaß der Expositionen gegenüber Schadstoffen in der Regel wesentlich niedriger als in der Arbeitswelt. Andererseits sind nicht nur gesunde Arbeitnehmer exponiert, sondern auch Kinder, Kranke, Schwangere und Alte, deren metabolische Kapazität im Vergleich zum gesunden Arbeitnehmer eingeschränkt sein kann. Unter diesen Umständen können interindividuelle Unterschiede in der metabolischen Kapazität, die bei einigen der untersuchten Sequenzvariationen auch den Faktor 100 überschreiten können, durchaus von Bedeutung sein. Diese möglichen Unterschiede sollten bei der Grenzwertfestsetzung angemessen berücksichtigt werden. Eine individuelle Risikovorhersage ist nach derzeitiger Einschätzung allerdings nicht möglich [45].
4.4 Monokausale Betrachtungsweise versus komplexe Autoregulation

Von einigen Ärzten werden die Erkenntnisse und Prinzipien bei Hochdosiseffekten (wie bei Arzneimitteln oder in der Arbeitswelt) unkritisch auf den umweltmedizinischen Niedrigdosisbereich übertragen. Einige willkürlich herausgegriffene Stoffwechseluntersuchungen dienen dabei zur Beurteilung der „Entgiftungskapazität" der Leber, und darauf fußend werden Therapieempfehlungen gegeben oder irreführende „Notfallausweise“ [46] ausgestellt. Diese Vorgehensweise wird anhand von methodisch meist unzureichenden Studien an „umweltmedizinischen Patienten“ begründet, über die im deutschen Sprachraum berichtet wird. Ihr liegt eine monokausale Denkweise zugrunde, wie sie schon aufgrund der außerordentlich komplexen „Biochemical Pathways“ nicht gerechtfertigt ist. Wenn man zusätzlich die hochvariablen Regulationsmechanismen auf der genetischen Ebene berücksichtigt, wird deutlich, weshalb solche monokausalen Ursache-Wirkungs-Beziehungen nur im Ausnahmefall und bei Hochdosiseinwirkung zu erwarten sind.

\subsection{Untersuchungen aus dem Bereich der ärztlichen Primärversorgung}

In einer Serie von Artikeln $[47,48,49]$ wurde über Bestimmungen von Enzympolymorphismen bei einem allmählich wachsenden Kollektiv von Patienten einer einzelnen Praxis berichtet. Über die Beobachtungszeit kamen wechselnde methodische Ansätze (zunächst Phänotypisierung mit unterschiedlichen Substraten, später Genotypisierung) zum Einsatz. Diese Publikationen stellen deskriptive Beobachtungen mit den eingeschränkten Möglichkeiten einer Arztpraxis dar. Verallgemeinernde Interpretationen dieser Befunde verbieten sich jedoch aufgrund der erheblichen methodischen Schwächen der erwähnten Studien. So fehlen z. B. eine präzise Krankheitsdefinition und eine Objektivierung der Diagnosen durch einen studienunabhängigen „Goldstandard“. Nach wissenschaftlichen Kriterien sind die postulierten Ursache-Wirkungs-Zusammenhän- ge daher, anders als in den Beiträgen dargestellt, nicht belegt, sondern können bestenfalls zur Hypothesenbildung herangezogen werden.

\section{Ethische Aspekte}

Molekularepidemiologische Untersuchungen zu Sequenzvariationen der FME leisten einen wichtigen Beitrag zur Aufklärung von Wirkungsmechanismen an Modellsubstanzen und können für die rationale Festlegung von Sicherheitsfaktoren bei der Risikobetrachtung herangezogen werden. Wenn die Ergebnisse solcher Untersuchungen für die individuelle Risikobetrachtung verwendet werden sollen, sind 2 Aspekte besonders zu berücksichtigen:

- die Vorhersagegenauigkeit, die bei monogenetischen Erbleiden hoch, aber beim Zusammenspiel mehrerer Gene und mehrerer Umweltfaktoren, wie im Fall der Sequenzvariationen der FME, begrenzt ist

- die soziale Implementierung, wie sie die Senatskommission für Grundsatzfragen der Genforschung der Deutschen Forschungsgemeinschaft 1999 in ihrer Stellungnahme zur Humangenomforschung und prädiktiven genetischen Diagnostik gefordert hat: $\mathrm{Zu}$ der angemessenen sozialen Implementierung gehört nicht nur die Bindung an medizinische Zwecke, sondern auch ein Arzt-Patienten-Verhältnis und damit eine qualifizierte Beratung. Der beratende Arzt muss der untersuchten Person die Bedeutung des Tests verständlich erklären. Auf bestehende Unsicherheiten bei der Ergebnisinterpretation oder der Prognose von Folgen muss ausdrücklich hingewiesen werden. Darüber hinaus ist es erforderlich, dass der untersuchten Person und ggf. der Familie Hilfestellung beim Umgang mit dem Testergebnis und dessen Folgen gegeben wird. Deshalb muss nicht nur die Qualität der Testverfahren, sondern auch die der sozialen Implementierung vor allem im Sinn einer ausführlichen genetischen Beratung sichergestellt werden [50]. 


\section{Zusammenfassung}

Fremdstoff-metabolisierende Enzyme spielen eine zentrale Rolle in der Auseinandersetzung des Organismus mit der Umwelt. Durch eine Vielzahl von Reaktionen können diese Enzyme selbst sehr lipophile Substanzen metabolisieren, sodass eine Elimination möglich wird. Diese Reaktionen werden in Phase-I- (Funktionalisierung) und Phase-II-Reaktionen (Konjugierung) unterschieden. Zum überwiegenden Teil sind die entstehenden Metaboliten biologisch nicht aktiv; es können aber sowohl im Phase-I- als auch im Phase-II-Metabolismus toxikologisch relevante Metaboliten entstehen. Die zentralen Phase-I-Enzyme sind die Cytochrome $\mathrm{P}_{450}$, die wichtigsten Phase-II-Enzyme sind die Glutathion-S-Transferasen, die N-Acetyltransferasen, die Sulfotransferasen und die UDP-Glukuronosyltransferasen. Jede dieser Enzymgruppen enthält mindestens 2 (N-Acetyltransferasen) oder mehr Enzyme, die sich durch die Expression in den Geweben, die Induzierbarkeit und die Substratspezifität unterscheiden können.

Seit einiger Zeit ist bekannt, dass zusätzlich auch noch Sequenzvariationen einen Einfluss auf die Fremdstoff-metabolisierenden Enzyme haben können. Die häufigsten Sequenzvariationen sind die Einzelnukleotidpolymorphismen, die - falls sie in den kodierenden oder regulatorischen Bereichen des Gens auftreten - eine Veränderung (Verminderung oder Erhöhung) der Enzymaktivität zur Folge haben können. Weiterhin wurden Deletionspolymorphismen, die beim homozygoten Träger zum vollständigen Ak-

\section{Anlage}

Von der Kommission benannter externer Sachverständiger PD Dr. Thomas Schulz (Federführung), Bundesinstitut für Risikobewertung, Thulalle 88-92, 14195 Berlin

Mitglieder der RKI-Kommission „Methoden und Qualitätssicherung in der Umweltmedizin"

Siehe Beitrag „RKI-Kommission Methoden und Qualitätssicherung in der Umweltmedizin" in diesem Heft. tivitätsverlust führen, und Multiplikationen, die eine Erhöhung der Enzymaktivität verursachen können, beschrieben. Es ist eine Vielzahl von Sequenzvariationen bekannt, und es ist mit der Entdeckung weiterer Variationen zu rechnen.

Sehr viele Studien haben Assoziationen zwischen den Sequenzvariationen und verschiedenen Erkrankungen untersucht: Hierbei wurden bislang vorwiegend maligne Erkrankungen ausgewählt. Es ist ein Trend erkennbar, dass nach initialer Veröffentlichung von signifikanten Assoziationen zwischen Sequenzvariationen und bestimmten Erkrankungen diese Zusammenhänge in Folgestudien sehr häufig nicht bestätigt werden konnten. Metaanalysen, die für eine Reihe dieser Assoziationen erarbeitet wurden, konnten nur für wenige Sequenzvariationen und bestimmte Erkrankungen eine Bestätigung liefern.

Vor diesem Hintergrund sind Untersuchungen der Sequenzvariationen Fremdstoff-metabolisierender Enzyme sinnvoll für wissenschaftliche Studien an geeigneten Kollektiven. Keinen Sinn hat zurzeit eine (individuelle) individualmedizinische Untersuchung, da es bisher kein Beispiel dafür gibt, dass eine Testung der heute bekannten Sequenzvariationen der Fremdstoff-metabolisierenden Enzyme konkrete Schutzmaßnahmen für ein Individuum gegenüber Umweltnoxen nahe legt. So erlaubt die Feststellung eines negativen GSTT1-Genotyps ebenso wenig die Diagnose einer „allgemeinen Entgiftungsschwäche" wie die Feststellung eines positiven GSTM1-Genotyps die Ausstellung eines Freibriefs für das Rauchen.

Abwertende Bezeichnungen, wie z. B. „ungünstige Mutationen“, sind also inhaltlich falsch und daher zu vermeiden. Ein kritikloser Gebrauch diagnostischer Daten aus solchen Tests kann unbegründete Ängste ebenso wie ein unbegründetes $\mathrm{Ge}$ fühl von Sicherheit hervorrufen, wodurch auch notwendige medizinische Maßnahmen unterbleiben könnten.

\section{Bewertung}

Die Anwendung von Genotypisierungsmethoden in der Umweltmedizin wird gemäß den „Grundsätzen der Bewertung von umweltmedizinischen Methoden“ [51] dieser Kommission in die $\mathrm{Ka}$ - tegorie IV eingestuft. Kategorie IV bedeutet, dass der Einsatz von Genotypisierungsmethoden für den klinisch-umweltmedizinischen Bereich nicht empfohlen werden kann, weil die Ergebnisse derzeit keine sinnvollen individual-medizinischen Aussagen ermöglichen. Insbesondere im umweltmedizinischen Niedrigdosisbereich von chemischen Noxen sind die bisher im erfahrungsmedizinischen Feld postulierten Zusammenhänge rein spekulativ und bieten daher keinen wissenschaftlich fundierten Erklärungs- oder gar Therapieansatz.

\section{Literatur}

1. Lander ES, Linton LM, Birren et al. (2001) Initial sequencing and analysis of the human genome. Nature 409:860-921

2. Seidegard J, Vorachek WR, Pero RW, Pearson WR (1988) Hereditary differences in the expression of the human glutathione transferase active on transstilbene oxide are due to a gene deletion. Proc Natl Acad Sci USA 85:7293-7297

3. Pemble SE, Schroeder KR, Spencer SR et al. (1994) Human glutathione S-transferase Theta (GSTT1): cDNA cloning and the characterization of a genetic polymorphism. Biochem J 300:271-275

4. Angerer J, Müller M (2004) Analyses of hazardous substances in biological material. Special issue: marker of susceptibility. Wiley-VCH, Weinheim

5. Schulz T (2003) Genetische Polymorphismen (Sequenzvariationen) von Fremdstoff-metabolisierenden Enzymen und ihre Bedeutung in der Umweltmedizin. Handbuch für Umweltmedizin, 27. Erg. Lfg. 10/03, Sektion III-2.2.11,1-20. Ecomed, Landsberg/Lech

6. Nebert DW (2000) Suggestions for the nomenclature of human alleles: relevance to ecogenetics, pharmacogenetics and molecular epidemiology. Pharmacogenetics 10:279-290

7. Bartsch H (2000) Studies on biomarkers in cancer etiology and prevention: a summary and challenge of 20 years of interdisciplinary research. Mutat Res 462:255-279

8. Wrighton SA, Stevens JC (1992) The human hepatic cytochromes P450 involved in drug metabolism. Crit Rev Toxicol 22:1-21

9. Hayes JD, Pulford DJ (1995) The glutathione Stransferase supergene family: regulation of GST and the contribution of the isoenzymes to cancer chemoprotection and drug resistance. Crit Rev Biochem Mol Biol 30:445-600

10. Dekant W, Koob M, Henschler D (1990) Metabolism of trichloroethene in vivo and in vitro evidence for activation by glutathione conjugation. Chem Biol Interact 73:89-101

11. Sherrat PJ, Hayes JD (2002) Glutathione $S$-transferases. In: loannides C (ed) Enzyme systems that metabolise drugs and other xenobiotics. Wiley, New York Weinheim, pp 319-352

12. Garte S, Gaspari L, Alexandrie AK et al. (2001) Metabolic gene polymorphism frequencies in control populations. Cancer Epidemiol Biomarkers Prev 10:1239-1248 
13. Engel LS, Taioli E, Pfeiffer R et al. (2002) Pooled analysis and meta-analysis of glutathione S-transferase $\mathrm{M} 1$ and bladder cancer: a HuGE review. Am J Epidemiol 156:95-109

14. Geisler SA, Olshan AF (2001) GSTM1, GSTT1, and the risk of squamous cell carcinoma of the head and neck: a mini-HuGE review. Am J Epidemiol 154:95-105

15. Cotton SC, Sharp L, Little J, Brockton N (2000) Glutathione S-transferase polymorphisms and colorectal cancer: a HuGE review. Am J Epidemiol 151:7-32

16. Benhamou S, Lee WJ, Alexandrie AK et al. (2002) Meta- and pooled analyses of the effects of glutathione S-transferase M1 polymorphisms and smoking on lung cancer risk. Carcinogenesis 23:13431350

17. Coughlin SS, Hall IJ (2002) Glutathione S-transferase polymorphisms and risk of ovarian cancer: a HuGE review. Genet Med 4:250-257

18. Meyer DJ, Coles B, Pemble SE et al. (1991) Theta, a new class of glutathione transferases purified from rat and man. Biochem J 274:409-414

19. Thier R, Wiebel FA, Hinkel A et al. (1998) Species differences in the glutathione transferase GSTT1-1 activity towards the model substrates methyl chloride and dichloromethane in liver and kidney. Arch Toxicol 72:622-629

20. Garnier R, Rambourg-Schepens MO, Müller A, Hallier $E$ (1996) Glutathione transferase activity and formation of macromolecular adducts in two cases of acute methyl bromide poisoning. Occ Environm Med 53:211-215

21. Chen H, Sandler DP, Taylor JA et al. (1996) Increased risk for myelodysplastic syndromes in individuals with glutathione transferase theta 1 (GSTT1) gene defect. Lancet 347:295-297

22. Atoyebi W, Kusec R, Fidler C et al. (1997) Glutathione S-transferase gene deletions in myelodysplasia. Lancet 349:1450-1451

23. Basu T, Gale RE, Langabeer S, Linch DC (1997) Glutathione S-transferase theta 1 (GSTT1) gene defect in myelodysplasia and acute myeloid leukaemia. Lancet 349:1450

24. Levy GN, Weber WW (2002) Arylamine acetyltransferases. In: loannides C (ed) Enzyme systems that metabolise drugs and other xenobiotics. Wiley, New York Weinheim, pp 441-457

25. Grant DM, Hughes NC, Janezic SA et al. (1997) Human acetyltransferase polymorphisms. Mut Res 376:61-70

26. Cartwright RA, Glashan RW, Rogers HJ et al. (1982) Role of $\mathrm{N}$-actyltransferase phenotypes in bladder carcinogenesis: a pharmacogenetic epidemiological approach to bladder cancer. Lancet II:842-846

27. Lewalter J, Mischke LW (1991) Empfehlungen zur arbeitsmedizinischen Prävention expositions- und dispositionsbedingter Arbeitsstoff-Beanspruchungen. Verhandlungen Dtsch Gesellschaft Arbeitsmed 31:135-139

28. Golka K, Prior V, Blaszkewicz M, Bolt HM (2002) The enhanced bladder cancer susceptibility of NAT2 slow acetylators towards aromatic amines: a review considering ethnic differences. Toxicol Lett 128:229-241

29. Marcus PM, Vineis P, Rothman N (2000) NAT2 slow acetylation and bladder cancer risk: a meta-analysis of 22 case-control studies conducted in the general population. Pharmacogenetics 10:115-122

30. Johns LE, Houlston RS (2000) N-acetyl transferase2 and bladder cancer risk: a meta-analysis. Environ Mol Mutagen 36:221-227
31. Vineis $P$, Marinelli D, Autrup H et al. (2001) Current smoking, occupation, $\mathrm{N}$-acetyltransferase- 2 and bladder cancer: a pooled analysis of genotype-based studies. Cancer Epidemiol Biomarkers Prev 10:1249-1252

32. Cascorbi I, Brockmöller J, Mrozikiewicz PM et al. (1996) Homozygous rapid arylamine $\mathrm{N}$-acetyltransferase (NAT2) genotype as a susceptibility factor for lung cancer. Canc Res 56:3961-3966

33. Lang NP, Chu DZ, Hunter CF et al. (1986) Role of aromatic amine acetyltransferase in human colorectal cancer. Arch Surg 121:1259-1261

34. Brockton N, Little J, Sharp L, Cotton SC (2000) $\mathrm{N}$-acetyltransferase polymorphisms and colorectal cancer: a HuGE review. Am J Epidemiol 151:846-861

35. Bock KW (2002) UDP-Glucuronosyltransferases. In: loannides C (ed) Enzyme systems that metabolise drugs and other xenobiotics. Wiley, New York Weinheim, pp 281-318

36. Glatt H (2002) Sulphotransferases. In: Ioannides $C$ (ed) Enzyme systems that metabolise drugs and other xenobiotics. Wiley, New York Weinheim, pp 353-439

37. Hayashi S, Watanabe J, Kawajiri K (1992) High susceptibility to lung cancer analyzed in terms of combined genotypes of P4501A1 and mu-class glutathione S-transferase genes. Jpn J Cancer Res 83:866-870

38. Rebbeck TR (1997) Molecular epidemiology of the human glutathione $\mathrm{S}$-transferase genotypes GSTM1 and GSTT1 in cancer susceptibility. Canc Epidem Biomark Prev 6:733-743

39. Vineis P, Schulte P, McMichael AJ (2001) Misconceptions about the use of genetic tests in populations. Lancet 357:709-712

40. Rothman N, Wacholder S, Caporaso NE et al. (2001) The use of common genetic polymorphisms to enhance the epidemiologic study of environmental carcinogens. Biochim Biophys Acta 1471:C1-C10

41. Ioannidis JPA, Ntzani EE, Trikalinos TA, Contopoulos-loannidis DG (2001) Replication validity of genetic association studies. Nat Genet 29:306-309

42. Garte $S$ (2001) Metabolic susceptibility genes as cancer risk factors: time for a reassessment? Cancer Epidemiol Biomarkers Prev 10:1233-1237

43. Schulz TG, Hallier E (1999) Die Bedeutung von genetischen Polymorphismen Fremdstoff-metabolisierender Enzyme in der Arbeitsmedizin. Arbeitsmed Sozialmed Umweltmed 34:307-314

44. Zinke E, Morun H (2000) Genetische Diagnostik und Arbeitsmedizin. Expertenanhörung der Enquete-Kommission „Recht und Ethik der modernen Medizin" des Deutschen Bundestages, Themengruppe 3 (Genetische Daten) 4.12.2000, Berlin

45. Schulz TG, Degen G, Foth H et al. (2002) Stellungnahme zur Bedeutung von genetischen Polymorphismen von Fremdstoff-metabolisierenden Enzymen in der Toxikologie. Umweltmed Forsch Praxis 7:232-246

46. Degen G, Foth H, Kahl R et al. (1999) Notfallausweis Glutathion-S-Transferasen - Stellungnahme der Sektion Toxikologie der DGPT. Umweltmed Forsch Prax 4:268

47. Fabig KR (1999) Glutathion-S-Transferase T1 und Multiple Chemikaliensensitivität (MCS). Umwelt Medizin Gesellschaft 11:226-232

48. Fabig (2002) Umweltgifte ohne genetische Antwort. Umwelt Medizin Gesellschaft 15:293-300
49. Fabig KR (2003) Polymorphismen in der Umweltmedizin. Erfahrungen und Indikationen. Umwelt Medizin Gesellschaft 16:103-111

50. DFG (1999) Humangenomforschung und prädiktive genetische Diagnostik: Möglichkeiten - Gren zen - Konsequenzen. Stellungnahme der Senatskommission für Grundsatzfragen der Genforschung der DFG. Eigenverlag, Bonn

51. Anonymus (2001) Grundsätze der Bewertung von umweltmedizinischen Methoden. Bundesgesundheitsblatt 5:519-522 